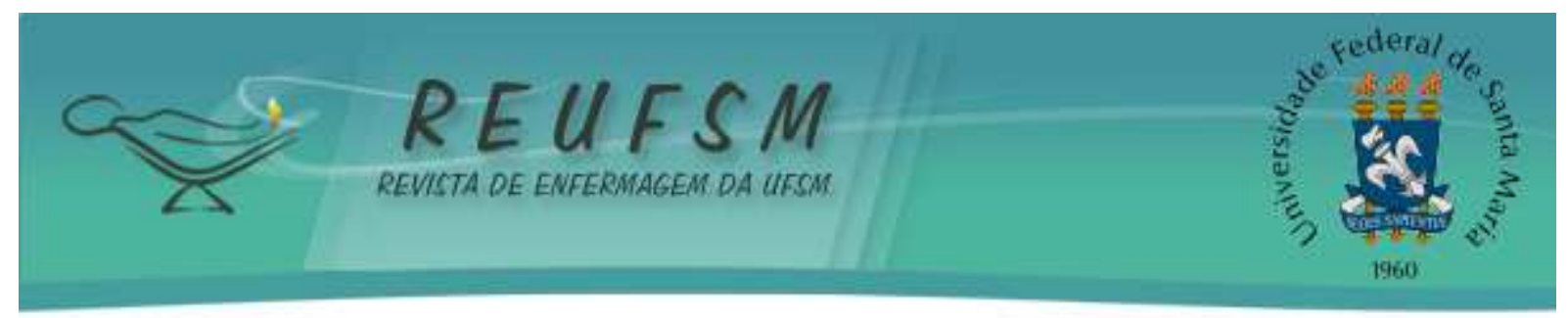

\title{
AVALIAÇÃO DE ESPERANÇA E RESILIÊNCIA EM PESSOAS EM TRATAMENTO HEMODIALÍTICO
}

\author{
EVALUATION OF HOPE AND RESILIENCE IN PEOPLE IN HEMODIALYTIC \\ TREATMENT
}

\section{EVALUACIÓN DE LA ESPERANZA Y LA RESILIENCIA EN PERSONAS EN TRATAMIENTO HEMODIALÍTICO}

\author{
Carine Ferreira ${ }^{1}$ \\ Maria Elena Echevarría Guanilo² \\ Denise Maria Guerreiro Vieira da Silva ${ }^{3}$ \\ Natália Gonçalves ${ }^{4}$ \\ Júlia Estela Willrich Boell ${ }^{5}$ \\ Barbara Letícia Dudel Mayer ${ }^{6}$
}

Doi: $10.5902 / 2179769230592$

RESUMO: Objetivo: avaliar a esperança manifestada em pessoas em tratamento hemodialítico e sua relação com a resiliência. Método: estudo transversal, em duas unidades de hemodiálise da região metropolitana de Florianópolis. Participaram 60 pessoas de janeiro a março de 2017. Utilizado instrumento de caracterização, Escala de Esperança de Herth adaptada e validada para a língua portuguesa, Escala de Resiliência de Connor - Davidson. Análise descritiva e coeficiente de correlação de Spearman. Resultados: correlação moderada significativa e positiva entre esperança e resiliência $(r: 0,470 ; p<0,01)$. Sexo feminino com valores médios maiores para esperança e resiliência (M: 44,72; DP: 3,23) e (M: 84,44; DP: 10,66). Os que se encontravam em lista de espera para o transplante obtiveram menores pontuações para esperança (M:42,75; DP: 4,76) e para resiliência (M:79,33; DP: 10,54). Conclusão: as pessoas em tratamento hemodialítico apresentam disposição à esperança e resiliência, o que sugere melhor adaptação ao tratamento.

Descritores: Enfermagem; Esperança; Resiliência psicológica; Insuficiência renal; Diálise renal

ABSTRACT: Aim: to evaluate the hope manifested by people undergoing hemodialysis and its relation with resilience. Method: cross-sectional study, in two hemodialysis units of the metropolitan region of Florianópolis. Sixty people participated in the study, from January to March 2017. Herth's Hope Scale, adapted and validated for the portuguese language and Connor - Davidson Resilience Scale were used as characterization instruments. Spearman's descriptive analysis and correlation coefficient. Results: moderate and positive correlation

\footnotetext{
1 Enfermeira, Universidade Federal de Santa Catarina. Florianópolis, Santa Catarina, Brasil. E-mail: carine2009@hotmail.com

${ }^{2}$ Enfermeira, Doutora, Universidade Federal de Santa Catarina. Florianópolis, Santa Catarina, Brasil. E-mail: elena_meeg@hotmail.com

${ }^{3}$ Enfermeira, Doutora, Universidade Federal de Santa Catarina. Florianópolis, Santa Catarina, Brasil. E-mail: denise_guerreiro@hotmail.com

${ }^{4}$ Enfermeira, Doutora, Universidade Federal de Santa Catarina. Florianópolis, Santa Catarina, Brasil. E-mail: nataliajbv@gmail.com

${ }^{5}$ Enfermeira, Doutora, Universidade Federal de Santa Catarina. Florianópolis, Santa Catarina, Brasil. E-mail: julia_estela8@hotmail.com

${ }^{6}$ Enfermeira, Mestre em Enfermagem, Universidade Federal de Santa Catarina. Florianópolis, Santa Catarina, Brasil. E-mail: barbaraldmayer@gmail.com
} 


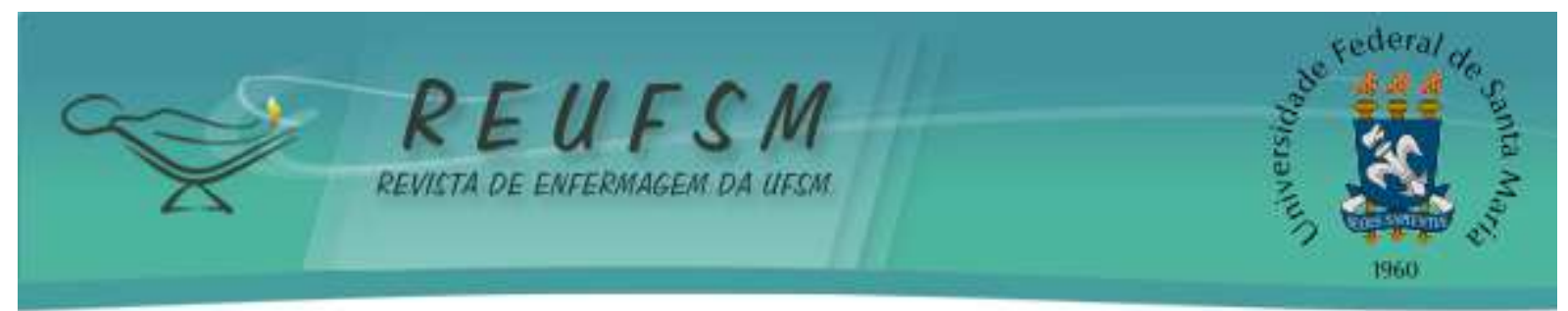

between hope and resilience ( $r$ : 0.470; $p<0.01)$. Females with higher mean values for hope and resilience (M: 44;72; SD: 3,23) and (M:84,44, SD: 10,66). Those on the waiting list for transplantation obtained lower scores for hope $(M: 42,75 ; S D: 4,76)$ and for resilience $(M$ : 79,33; SD: 10,54). Conclusion: people on hemodialysis have a disposition to hope and resilience, which suggests a better adaptation to treatment.

Descriptors: Nursing; Hope; Resilience psychological; Renal insufficiency; Renal dialysis

RESUMEN: Objetivo: evaluar la esperanza revelada en personas en tratamiento hemodialítico y su relación con la resiliencia. Método: estudio transversal, realizado en dos unidades de hemodiálisis de la región metropolitana de Florianópolis. Participaron 60 personas entre enero y marzo de 2017. El instrumento de caracterización fue Herth Esperanza Escala, adaptado y validado para el idioma portugués, Connor - Davidson Resiliencia Escala. Análisis descriptivo y coeficiente de correlación de Spearman. Resultados: correlación moderada significativa y positiva entre esperanza y resiliencia $(r: 0,470 ; p<0,01)$. El sexo femenino con valores medios mayores para la esperanza y la resiliencia $(M: 44,72 ; D P: 3,23)$ y (M: 84,44; DP: 10,66). Los que se encontraban en lista de espera para el trasplante obtuvieron menores puntuaciones para la esperanza (M: 42,75; DP: 4,76) y para resiliencia (M: 79,33; DP: 10,54). Conclusión: las personas en tratamiento hemodialítico presentan predisposición a la esperanza y a la resiliencia, lo que sugiere mejor adaptación al tratamiento.

Descriptores: Enfermería; Esperanza; Resiliencia psicológica; Insuficiencia renal; Diálisis renal

\section{INTRODUÇÃO}

As doenças crônicas não transmissíveis (DCNT) representam um importante problema de saúde pública mundial, estimando-se as mesmas são responsáveis por 16 milhões de mortes prematuras (antes dos 70 anos), odos os anos no mundo. ${ }^{1}$ No Brasil estima-se que, aproximadamente, $72 \%$ das mortes são causadas pelas DCNT.2 São diversas as doenças categorizadas como DCNT, cada qual com suas especificidades de prevenção, diagnóstico e tratamento e, entre elas, está a doença renal crônica (DRC). Pessoas com DRC representam hoje, $10 \%$ da população mundial, estimando-se a existência de mais de dois milhões de brasileiros com algum grau de disfunção renal, sendo que mais de $70 \%$ desconhecem a sua condição. ${ }^{3}$ Dados como esses colocam a DRC como problema a nível mundial de saúde pública, assim como um importante indicador na área da saúde, visto que, a mesma compreende expressivo gasto para o Sistema Único de Saúde (SUS) relacionado ao seu tratamento, ao contingente de pessoas jovens que evoluem para cronificação da doença, e ainda, pelas mortes prematuras. ${ }^{4}$

A falência renal compreende a redução da capacidade dos rins em remover os produtos de degradação metabólica do corpo e, consequentemente, altera suas funções reguladoras. ${ }^{5} \mathrm{O}$ 


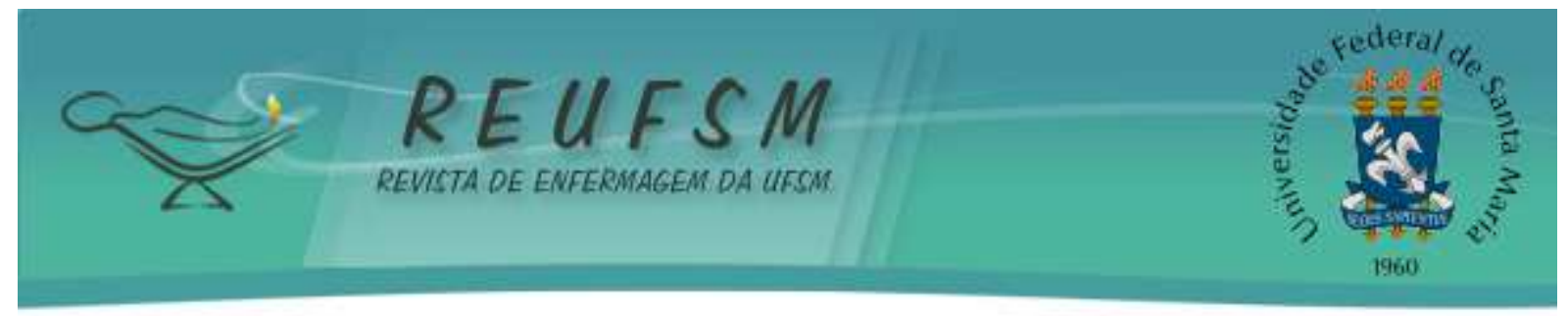

tratamento pode ser o conservador, a diálise peritoneal, hemodiálise (HD) ou transplante. Atualmente a opção mais utilizada, seja na fase aguda ou crônica da doença, é a HD. Para isso, torna-se necessária a inserção de um cateter implantável (temporário ou permanente) ou a realização de uma fístula arteriovenosa como via de acesso para a realização da HD. Consequentemente, a pessoa vai necessitar se deslocar do domicílio até o centro de tratamento, com uma frequência de duas a três vezes por semana, permanecendo conectado ao equipamento de diálise por um tempo aproximado de três a quatro horas, dependendo das necessidades individuais. ${ }^{6}$ Deste modo, embora a opção por este tratamento represente a possibilidade de vida, este envolve adaptações importantes, uma vez que o seu caráter de cronicidade ocasiona impacto nas esferas da vida pessoal e profissional do indivíduo.

A descoberta da doença afeta tanto a pessoa quanto a sua família e a onvivência com a nova condição de saúde provoca mudanças significativas nas atividades de vida diária, exigindo adaptações que lhe permitam viver uma vida mais saudável. ${ }^{7}$

As modificações implicam na realização de cuidados como adequação da dieta alimentar, prática de atividades físicas, uso de medicamentos, controle e monitoramento da saúde por meio de exames, dentre outras mudanças incorporadas para uma adesão efetiva. ${ }^{8}$ Assim, é esperado que os pacientes vivenciem sentimentos de desesperança. Manter a esperança frente à doença crônica é um processo contínuo e essencial, uma vez que é um recurso valioso no processo de enfrentamento dessa condição. Possui efeito benéfico para a saúde das pessoas, ao contribuir para a capacitação da mesma ao lidar com situações de crise, para manutenção da qualidade de vida, determinação de objetivos saudáveis e para a promoção da saúde. ${ }^{9}$

Outro conceito que pode estar atrelado à situação vivenciada por pessoas que realizam HD é a resiliência. Apesar das diversas definições, o conceito de resiliência converge para a adaptação positiva frente à adversidade, situação com a qual as pessoas em HD lidam constantemente. ${ }^{10}$ Nesse contexto, a esperança e a resiliência também estão associadas como ferramentas de apoio e fortalecimento para o enfrentamento das dificuldades no cotidiano, relacionadas à sua condição de saúde.

Considerando a pouca evidência acerca da relação esperança e resiliência de pessoas em $\mathrm{HD}^{11}$ buscou-se desenvolver este estudo respondendo à questão de pesquisa: qual a manifestação de esperança e resiliência em pessoas que se encontram em tratamento hemodialítico, há relação 


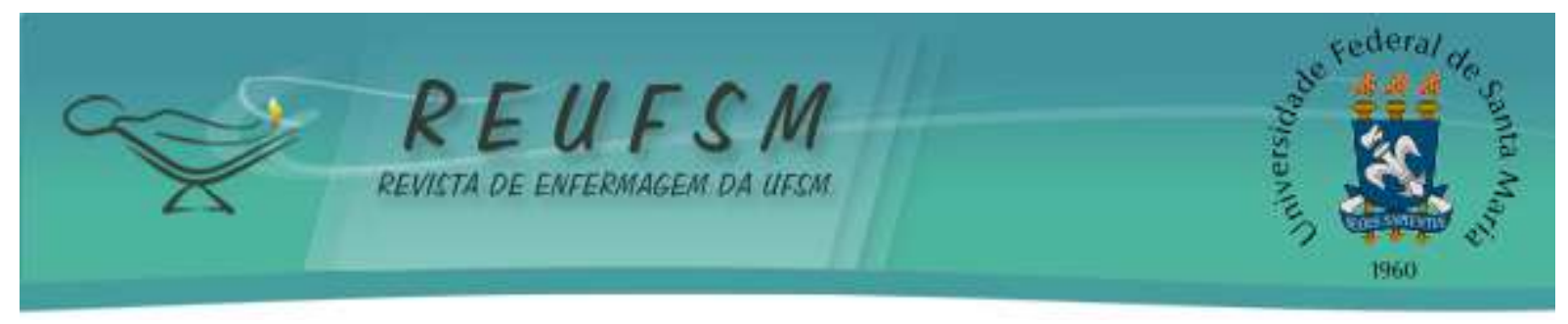

dessas variáveis? Dessa forma, o objetivo do estudo foi avaliar a esperança manifestada em pessoas em tratamento hemodialítico e sua relação com a resiliência.

\section{MÉTODO}

Trata-se de um estudo do tipo observacional, transversal e abordagem quantitativa de análise dos dados, realizado com pessoas atendidas no serviço de HD de um hospital público de grande porte (local 1) e um centro de $\mathrm{HD}$, de caráter público-privado (local 2). Participaram do estudo, adultos com idade igual ou superior a 18 anos que se encontravam realizando HD há, pelo menos, dois meses.

Foram excluídas as pessoas que não atingiram a pontuação mínima ( 20 pontos) no MEEM; que apresentaram instabilidade clínica, tais como complicações que gerassem necessidade de hospitalização. Para o cálculo amostral se tomou como base a população de 160 pessoas que eram atendidas nos dois centros de hemodiálises pesquisados, dos quais 80 atendiam aos critérios de inclusão e exclusão. Também se considerou uma prevalência de deficiência de 50\%, nível de significância de $90 \%$ e erro amostral de 5\%. Assim, a amostra foi calculada para 62 pessoas realizando hemodiálise, das quais duas não atenderam a pontuação mínima do MEEN. A inclusão dos participantes foi de forma sequencial, seguindo a ordem de atendimento, no período de janeiro até março de 2017.

Os participantes foram convidados a participar do estudo durante a primeira hora da sessão de hemodiálise, sendo informados sobre os objetivos e benefícios do estudo e aos interessados na participação, foi solicitada assinatura do o termo de consentimento livre esclarecido (TCLE), e imediatamente após a entrevista era iniciada. Foram aplicados três instrumentos de coleta de dados, sendo um instrumento de caracterização dos participantes, um questionário para avaliar esperança e um para avaliação de resiliência.

O instrumento de caracterização foi elaborado pelas autoras, a partir do objetivo do estudo o qual foi direcionado para a obtenção dos dados sociodemográficos. As variáveis foram: sexo, idade, data de nascimento, procedência, estado civil, escolaridade, religião, hábito de rezar, renda individual e familiar, tempo de doença renal crônica, tempo e tipo de diálise, tempo de transplante renal, se possui alguém que o ajude no tratamento e se o mesmo se encontra cadastrado em lista de espera para o transplante e o porquê. 


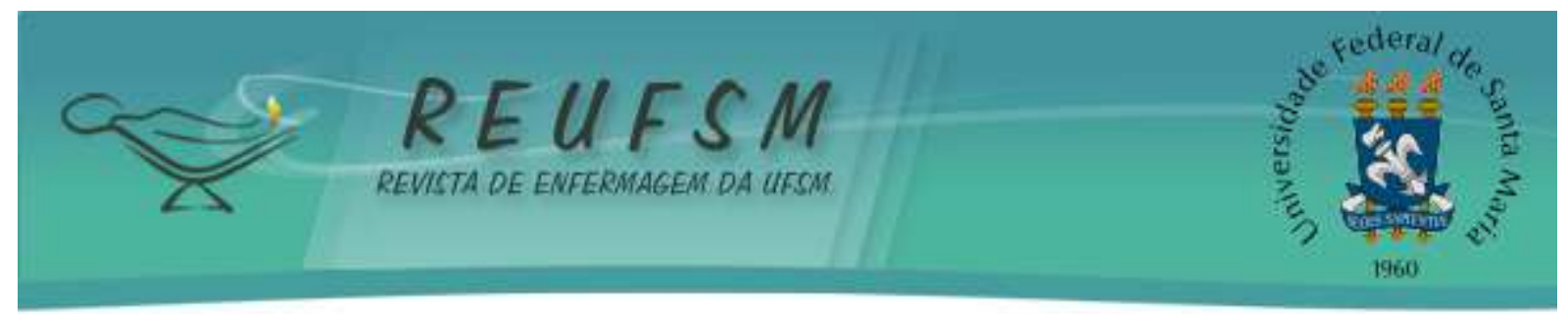

Para avaliar a esperança foi utilizada a Escala de Esperança de Herth (EEH) adaptada e validada para a língua portuguesa sendo que, a escala originou-se do instrumento americano Herth Hope Index. ${ }^{12}$ A confiabilidade foi verificada através da análise da consistência interna demonstrada pelos resultados satisfatórios do coeficiente Alpha de Cronbach total de 0,834. O instrumento apresenta 12 afirmações com respostas em escala do tipo Likert com escores de 1 a 4 para cada uma delas e as pontuações são somadas ao final do questionário. As respostas possíveis e seus respectivos valores são: $1=$ discordo completamente; 2 = discordo; $3=$ concordo; 4 = concordo completamente. Os itens três e seis da escala apresentam escores invertidos. O valor obtido pode variar de 12 a 48 pontos, com pontuações mais altas representando maior esperança. ${ }^{13}$ Para o cálculo, deve-se considerar que a afirmação do item três e do seis apresentam escores invertidos.

A resiliência foi avaliada utilizando a Escala de Resiliência de Connor - Davidson (ERCD),${ }^{14}$ sendo que após o contato com os autores foi autorizado o uso e disponibilizada a versão adaptada e validada para a população brasileira. É composta por 25 itens, cada qual avaliado em uma escala de 0 a 4 pontos com escores mais altos refletindo maior resiliência: $0=$ nem um pouco verdadeiro; 1 = raramente verdadeiro; 2 = às vezes, verdadeiro; $3=$ frequentemente verdadeiro; 4 = quase, sempre verdadeiro.

A escala foi avaliada com relação à confiabilidade e à validade (consistência interna, teste/ reteste, validade convergente e validade discriminante) e ao fator estrutural e foi considerada como tendo boas propriedades psicométricas, permitindo distinguir entre pessoas com maior ou menor resiliência, a pontuação pode varias de 0 a 100 pontos.

Os dados foram organizados e processados no Statistical Package for the Social Sciences (IBM SPSS v.20), sendo obtidas análises estatísticas descritivas e o Coeficiente de correlação de Spearman. Para a análise dos valores dos coeficientes de correlação, foi seguida a classificação proposta por Ajzen e Fishbein: valores menores que 0,30 correlações fracas e de pouca aplicabilidade clínica, mesmo quando estatisticamente significantes; valores entre 0,30 e 0,50 , correlações moderadas e acima de 0,50 , correlações fortes. Também foi obtido o alpha de cronbach das escalas aplicadas.

O projeto foi aprovado pelo Comitê de Ética em Pesquisa com Seres Humanos no dia três de dezembro de 2016, sob o parecer 1.849.031, bem como anuência do hospital e aprovação do centro de HD. 


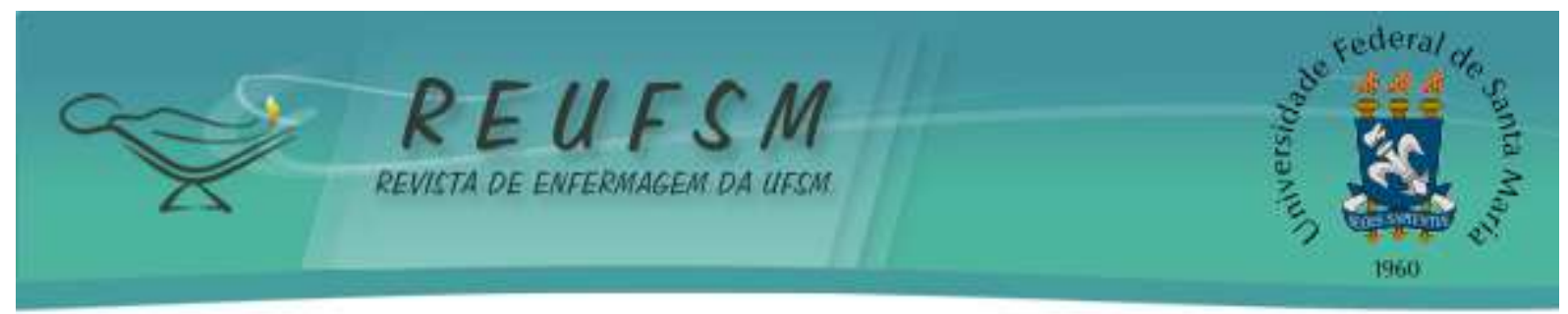

\section{RESULTADOS}

Dos integrantes da pesquisa 58,3\% eram do sexo masculino, jovens (M: 51,53; DP: 15,01), 55\% se declararam católicos, média de 13,07 anos de estudos (DP: 6,49), renda individual média de R \$ 1.942,9492 (DP: 1.853,05), sendo que mais da metade dos participantes referiram ser aposentados. Os participantes realizavam HD há, aproximadamente, 2 anos e 5 meses (DP: 2,55), sendo que destes, $10 \%$ permaneceram um período transplantados sem hemodiálise, com uma duração média de um ano e nove meses (Tabela 1).

A aplicação da Escala de Esperança apresentou pontuação média de 44,06 (DP: 4,11). A partir da possibilidade da pontuação de cada item (variando de um a quatro pontos), destaca-se que dos doze itens avaliados, três apresentaram expressiva representatividade negativa para as pessoas, sendo estes: "Eu tenho medo do meu futuro" (M: 2,86; DP: 2,86), "Eu me sinto muito sozinho (a)" (M: 3,15; DP: 1,23) e "Eu me sinto muito forte" (M: 3,63; DP: 0,66).

A Escala de Resiliência apresentou uma pontuação global de 83,91 (DP: 11,54). Dos 25 itens dispostos na escala, os três que tiveram expressiva representatividade negativa para as pessoas foram, "Eu gosto de desafios" (M: 2,62; DP:1,54), "Se for necessário eu consigo tomar decisões difíceis ou desagradáveis que afetem outras pessoas" (M:2,37; DP: 1,64) e "Ter que lidar com situações estressantes me faz sentir mais forte" (M:2,67; DP:1,42), podendo variar de zero a quatro pontos.

Em relação ao estudo da correlação entre os valores obtidos para Esperança e Resiliência, obteve-se correlação estatisticamente significante, moderada e positiva (r: 0,470; $p<0,01$ ) e um alpha de 0,794 (Tabela 2).

Tabela 1 - Distribuição das variáveis sociodemográficas e clínicas dos participantes do estudo. Florianópolis, 2017.

$\begin{array}{lccc}\text { Variáveis } & \text { n. } & \text { Média } & \text { Desvio Padrão (DP) } \\ \text { Idade } & & 51,53 & 15,01 \\ \text { Sexo } & & & \\ \quad \text { Feminino } & 25 & & \\ \quad \text { Masculino } & 35 & & \\ \text { Estado civil } & & & \\ \quad \text { Casado/União estável } & 18 & & \\ \quad \text { Solteiro } & 28 & & \\ \quad \text { Viúvo } & 6 & & \\ \quad \text { Divorciado } & 8 & \\ \text { Escolaridade } & & \\ \quad \text { Fundamental incompleto } & 21 & \\ \quad \text { Fundamental completo } & 15 & \\ \quad \text { Ensino médio } & 18 & \end{array}$




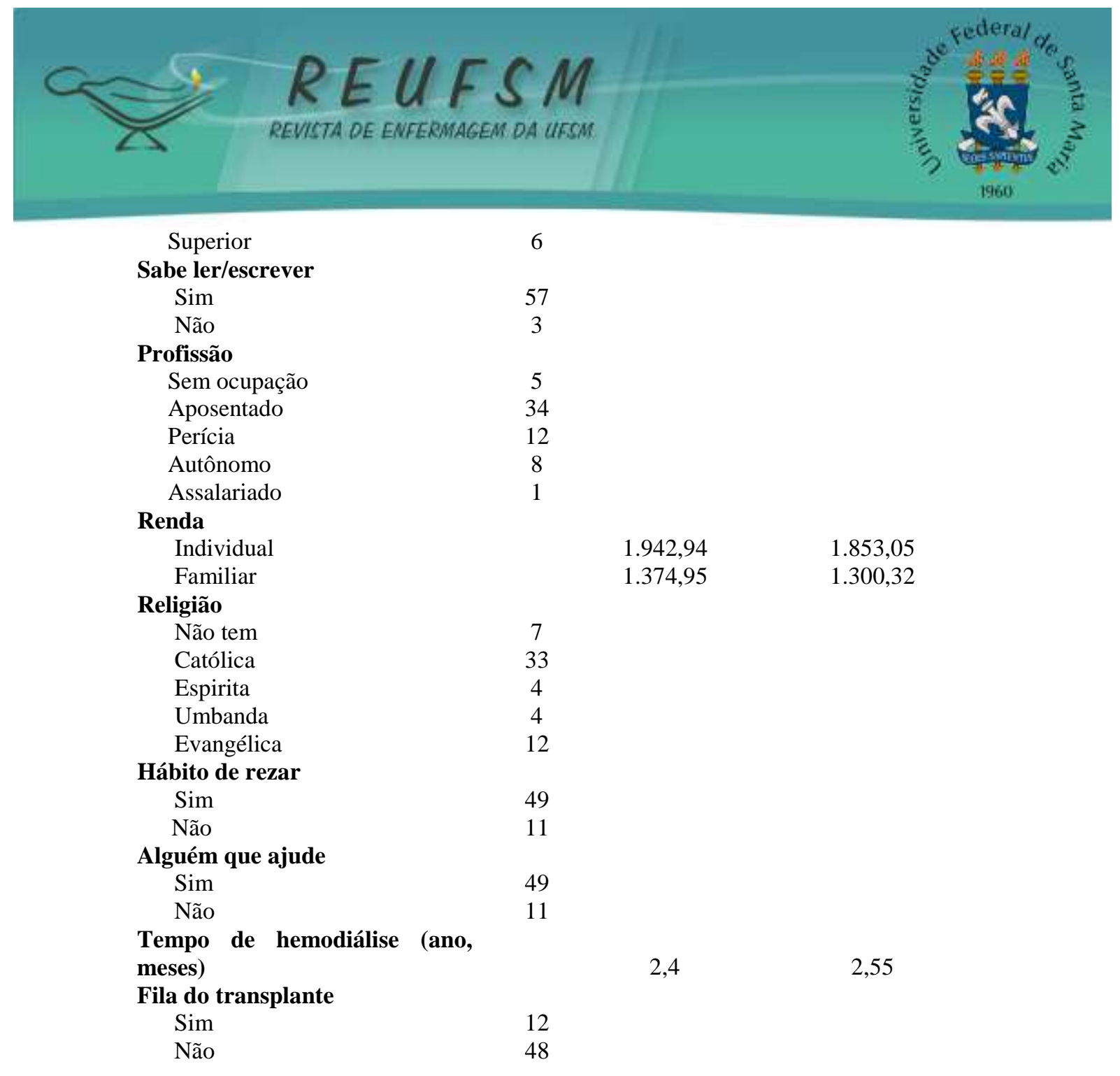

Tabela 2 - Distribuição dos valores de Esperança e Resiliência e sua correlação. Florianópolis, 2017.

$\begin{array}{cccccccr} & \text { Média } & \begin{array}{c}\text { Desvio padrão } \\ (\mathrm{DP})\end{array} & \text { Mediana } & \text { Mínimo } & \text { Máximo } & \text { Correlação }(\mathrm{r}) & \text { Alfa }(\alpha) \\ \text { EEH } & 44,06 & 4,11 & 45,00 & 25 & 48 & 0,470^{* *} & 0,695 \\ \text { ERCD } & 83,91 & 11,74 & 85,50 & 29 & 100 & & 0,825\end{array}$

** A correlação é significativa no nível 0,01 .

Quando analisadas as pontuações das escalas de esperança e resiliência segundo o sexo, é possível identificar pontuações maiores para as mulheres, tanto em esperança (M: 44,72; DP: 3,23) quanto em resiliência (M: 84,44; DP: 10,66), quando comparados com as pontuações dos homens (M: 43,70; DP: 4,62 e M: 83,54; DP: 12,59, respectivamente) (Figura 1). 

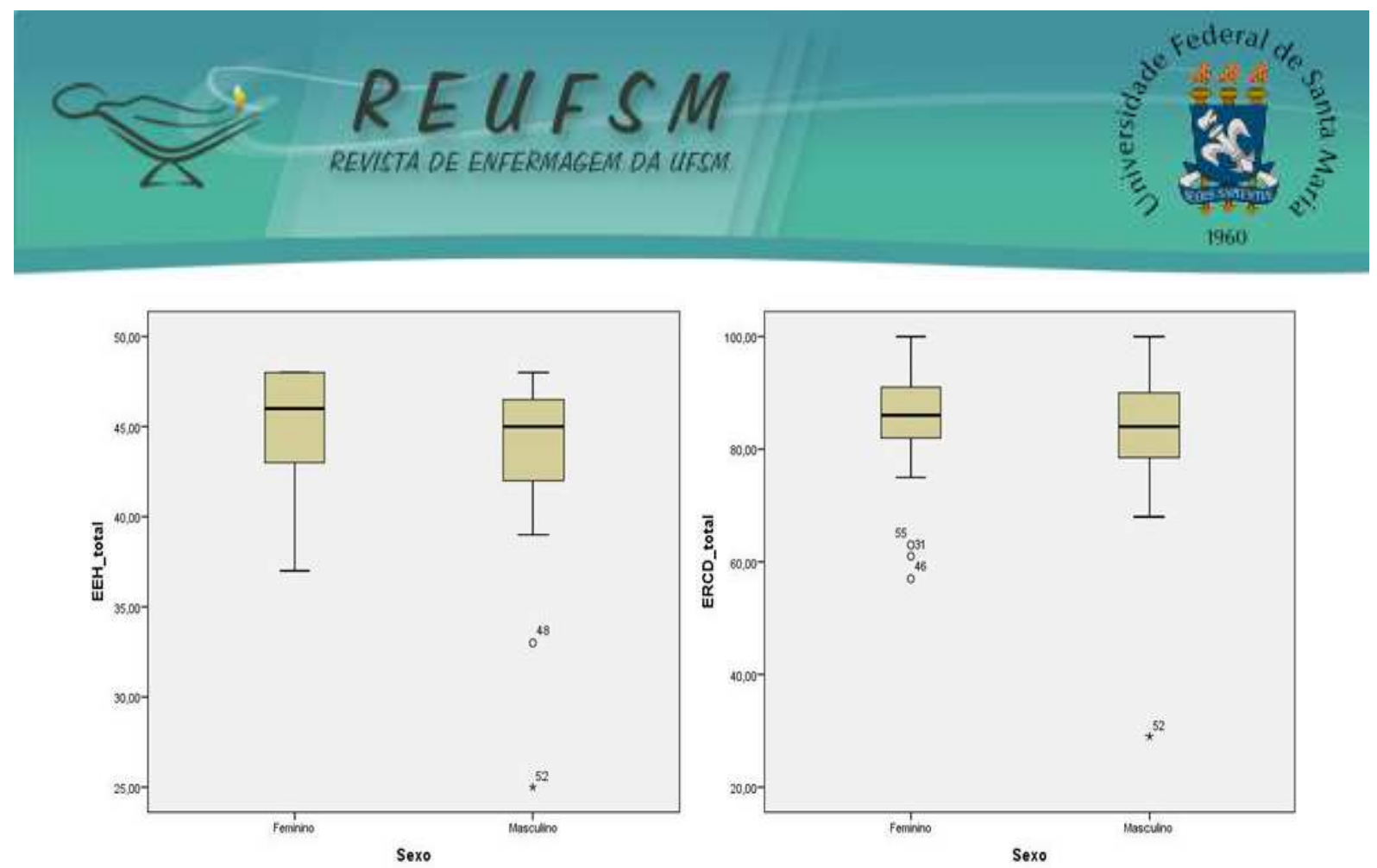

Figura 1 - Esperança e resiliência em pacientes renais crônicos em tratamento hemodialítico na comparação entre os sexos.

Quando considerada as pontuações em relação a ter alguém que ajude no tratamento, as pontuações das duas escalas foram maiores para as pessoas que relataram ter alguém que o ajudava nos cuidados necessários para realizar o tratamento. Assim, pessoas que tinham ajuda apresentaram uma média de esperança de 44,71 (DP: 2,81), e de resiliência de 85,81 (DP: 8,84), e pessoas que não contavam com alguém apresentaram pontuação de 41,18 (DP: 7,11) e de 75,45 (DP: 18,47), respectivamente (Figura 2).
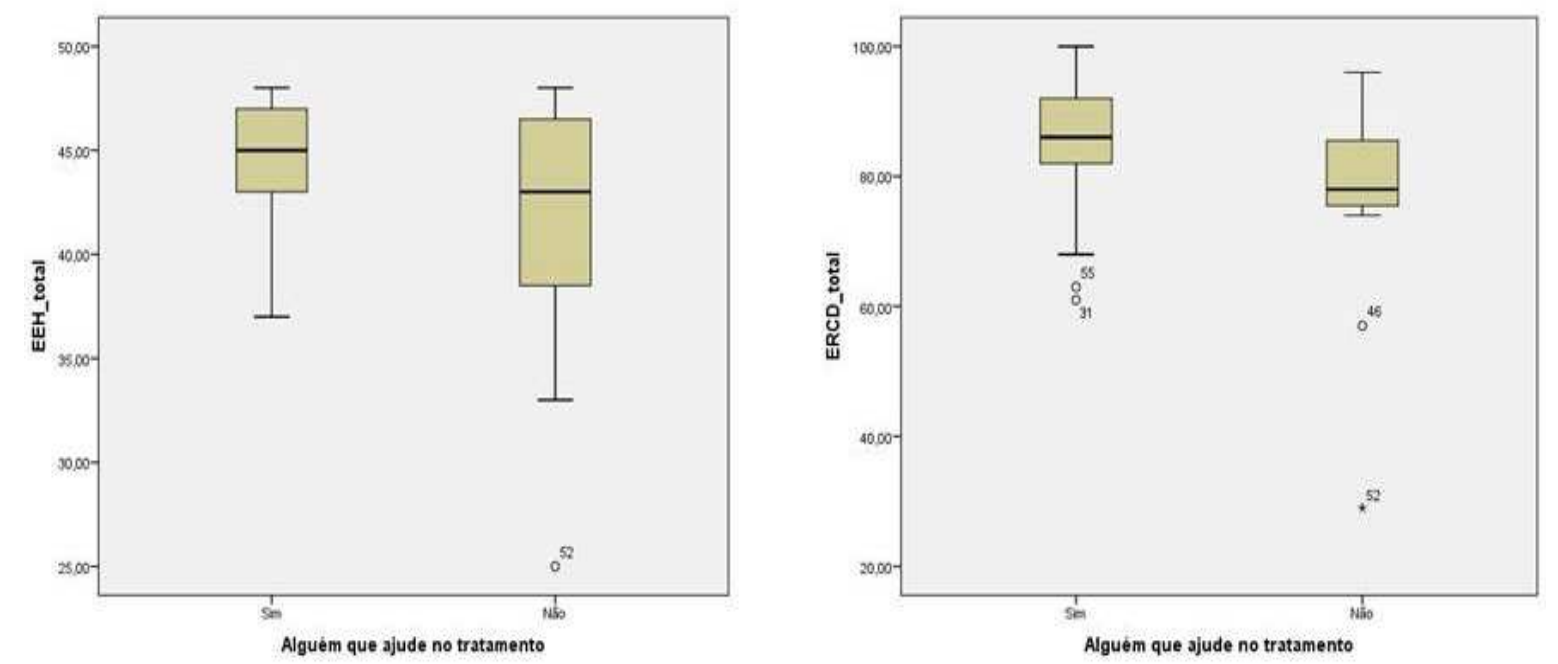

Figura 2 - Esperança e resiliência em pacientes renais crônicos em tratamento hemodialítico, na comparação entre quem possui e quem não possui alguém que ajude no tratamento. 


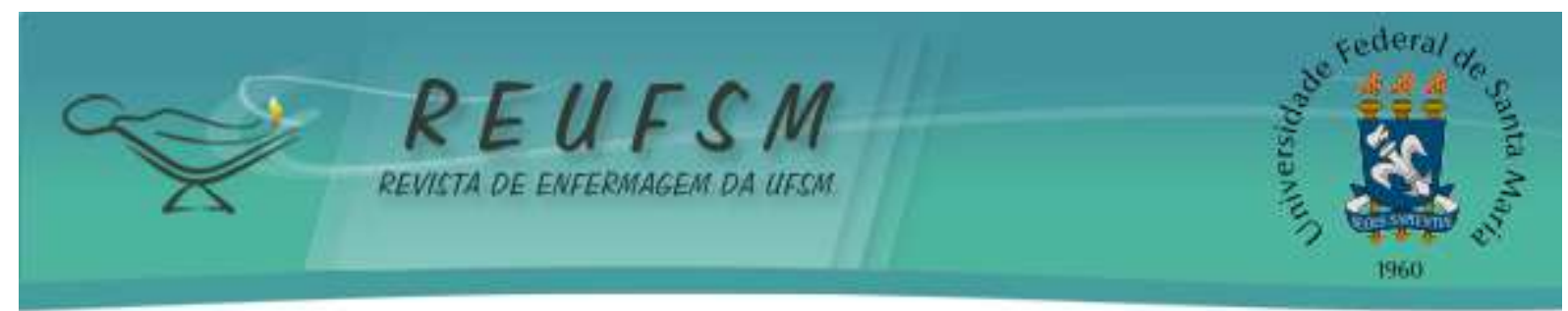

Em relação a estarem cadastradas em lista de transplante de rim, as pessoas que se encontram em lista de transplante apresentaram menores pontuações para esperança e resiliência, em relação aos que não estavam. Apresentaram pontuação média de esperança de 42,75 (DP: 4,76) e de resiliência de 79,33 (DP: 10,54). Ainda, foi possível observar que as pessoas que se encontravam em lista de espera para transplante eram pessoas mais jovens, em idade produtiva. Aquelas que não estavam em lista de espera, apresentaram valor médio de esperança de 44,39 (DP: 3,91) e de resiliência de 85,06 (DP: 11,84), respectivamente (Figura 3).
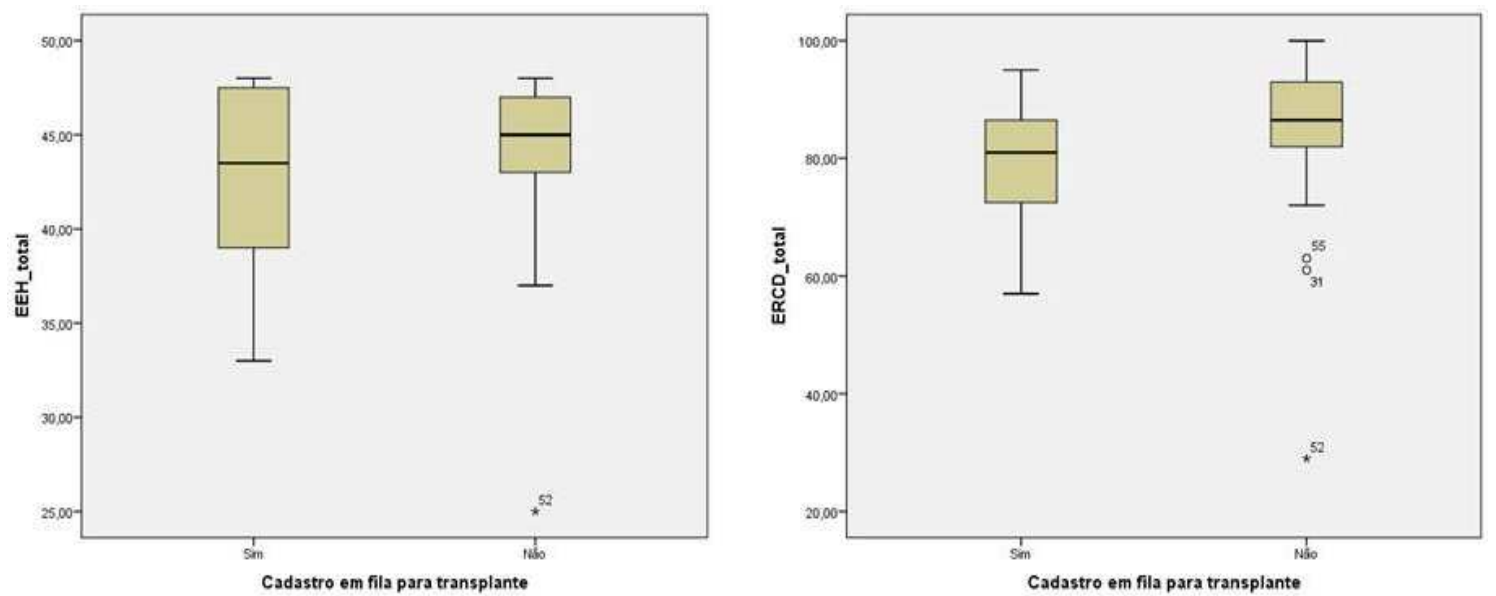

Figura 3 - Esperança e resiliência em pacientes renais crônicos em tratamento hemodialítico, na comparação entre quem está e não está em lista de transplante.

A respeito de estar ou não cadastrado em lista de espera para o transplante, a decisão que representou a maior parte dos participantes foi a de não estar na lista de espera para transplante renal, relatando como principal razão o fato desse tratamento exigir muitos cuidados; ter a possibilidade de complicações; se considerarem frágeis fisicamente e emocionalmente; ter risco de morte; a possibilidade de reincidir para HD e ser uma opção de tratamento que pode não ser definitiva.

\section{DISCUSSÃO}

O perfil dos participantes deste estudo - sexo masculino, jovens, católicos, média de 13,07 anos de estudos, aposentados - veio ao encontro da literatura. ${ }^{8} \mathrm{Na}$ Escala de Esperança, os itens acerca do medo em relação ao futuro e de sentir-se sozinho evidenciaram valores menores, o que pode estar relacionado ao fato de uma condição crônica gerar situações de dependência em relação à família e aos serviços de saúde, com necessidade de importantes 


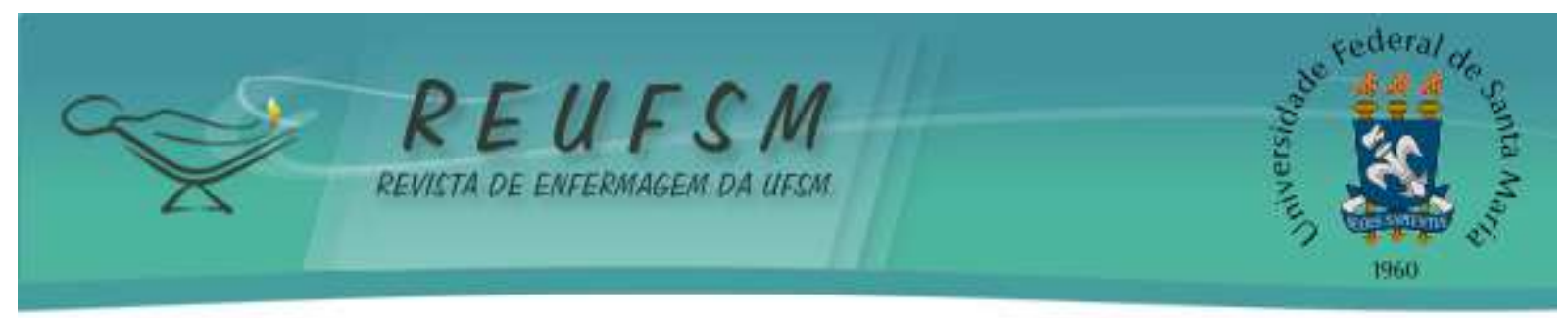

mudanças na rotina individual e familiar em decorrência do tratamento, gerando sobrecarga familiar. Os valores evidenciados na presente pesquisa na Escala de Esperança quando comparada com outros estudos, pode ser considerada um valor elevado. Foi encontrado, no contexto brasileiro, estudo com objetivo avaliar o nível de esperança em 50 pessoas idosas em HD, sendo identificados uma média de esperança de 36,20 (DP: 2,90$){ }^{8}$

Em comparação com a atual, observou-se que, independentemente de estar ou não na lista de transplante, o nível de esperança apresentado pelos idosos foi mais baixo, refletindo uma resposta menor em relação à manifestação de esperança. Ainda, é importante destacar que a amostra do presente estudo, não se restringiu a idosos, incluindo também jovens e adultos. $\mathrm{O}$ outro estudo foi realizado com três grupos de pessoas com câncer, diabetes e seus acompanhantes (familiares ou cuidadores), os quais obtiveram um valor de esperança de 41,57, 40,46 e 40,46, respectivamente, que são valores inferiores à média obtida na presente pesquisa. ${ }^{15}$

No âmbito internacional foram encontrados estudos relacionados à esperança em pessoas em condição crônica, incluindo úlcera venosa crônica, Diabetes tipo 2 e em pessoas com câncer. ${ }^{16-18}$ Dentre estes estudos, observa-se que o nível de esperança dos participantes do presente estudo foi superior ao encontrado na maioria das pesquisas com pessoas acometidas por outras doenças crônicas, que variou entre 16,5 e 34,89.

Quando analisados os itens com pontuações mais baixas da Escala de Resiliência, os aspectos relacionados às estratégias que as pessoas têm para a vivência de situações geradoras de importantes mudanças, tais como lidar com desafios e situações consideradas estressantes e tomar decisões que afetem a sua saúde e a organização de vida de outras pessoas levam a manifestações de resiliência mais baixa. Ações de cuidados em etapas precoces da doença podem levar ao desenvolvimento de melhores formas de viver em uma condição crônica.

Em relação à resiliência, estudo com a aplicação da ERCD foi identificado uma média de resiliência igual a 79,8 (DP: 12,9) para pessoas com DM, maior do que as pessoas com DRC, que apresentaram média de resiliência de 67,5 (DP:15,4). ${ }^{19}$ Ainda assim, esses valores são mais baixos que os apresentados pelo presente estudo (M: 83,91; DP: 11,74).

Estudo que utilizou a Escala de Wagnild e Young (1993) para avaliação da resiliência em pessoa em HD, identificou uma média de 131,3 pontos (DP: 20,06), cuja pontuação poderia varia de 25 até $175 .{ }^{20}$ Outra pesquisa que utilizou a mesma escala, constatou que $61 \%$ 


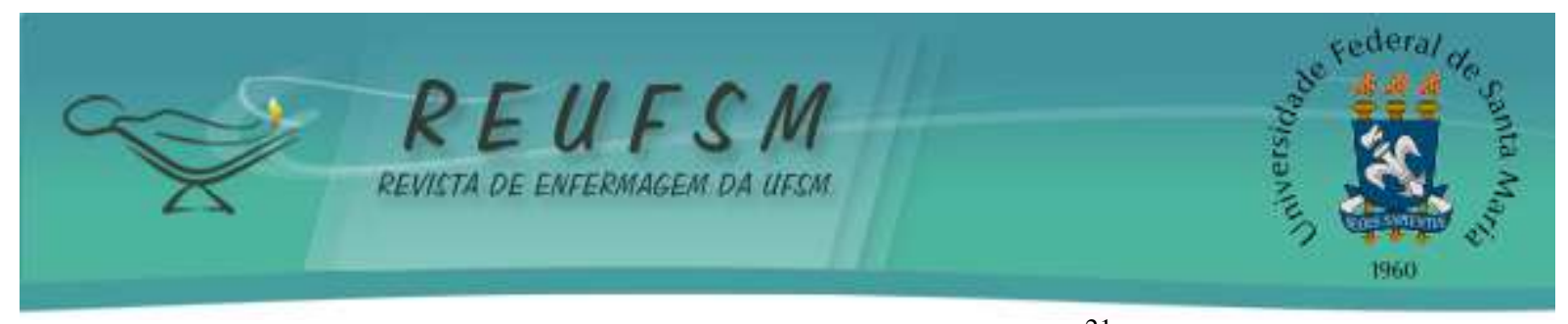

dos pacientes apresentaram importante tendência à resiliência. ${ }^{21}$ Assim como no presente estudo, o nível de resiliência apresentado foi considerado elevado.

Ao analisar as variáveis esperança e sexo, não foram encontrados estudos específicos para discussão, entretanto, com relação à resiliência segundo sexo, pesquisadores encontraram que o sexo masculino apresenta melhores pontuações para resiliência. ${ }^{22}$ A esse respeito, na amostra estudada, foi possível identificar maiores pontuações de resiliência para as mulheres, entre as pessoas que contam com alguém para ajudá-lo com os cuidados e para as pessoas que se encontravam na lista de espera para transplante renal.

Em relação a vivenciar a DRC e poder contar com alguém que o ajude nesse cuidado, autores relatam que a HD pode se tornar suportável e rotineira para os pacientes, mesmo que o comprometimento dos aspectos emocionais e sociais estejam afetados. Nesse sentido, as relações sociais e familiares são consideradas influenciadoras na qualidade de vida do ser humano, uma vez que relações harmônicas são de fundamental importância para a manutenção da saúde mental. ${ }^{23}$ A família, por ser uma unidade de cuidado na qual seus membros possuem laços afetivos, torna-se importante na vida da pessoa com doença crônica, favorecendo a expressão e vivência o sentimento de esperança e de resiliência da mesma.

A partir dos resultados apresentados, principalmente entre os que relataram ter alguém que o ajude no cuidado, identifica-se a importância das pessoas se sentirem apoiadas no decorrer do processo da doença, uma vez que estas pessoas apresentaram os valores mais altos de esperança e resiliência, quando comprados com os que relataram não ter alguém que os ajudasse no cuidado da sua saúde. Nesse sentido, em relação ao estado civil, a maioria dos indivíduos que relatou ser solteiro, também relatou contar com alguém que o ajudava no cuidado.

Literatura evidencia que a percepção de pessoas em HD fora da lista de espera é de se sentirem seguros e adaptados às condições da vida em HD, de modo a permanecer nesta terapia, mesmo possuindo indicação para transplante. ${ }^{24}$ Assim, os mesmos justificam a sua opção pela necessidade de medicações, questões financeiras, burocracia para ingressar na lista, incertezas e experiências negativas do transplante; possibilidade de complicações e de retorno à HD e medo da morte. Entretanto, vale salientar que apesar dos motivos elencados pelas pessoas serem relevantes para o não ingresso em lista de espera, não se descarta a necessidade de serem informados e esclarecidos sobre esta terapêutica. Já entre os, sentimentos expressados pelas pessoas que esperam pelo transplante, destacam-se: esperança, 


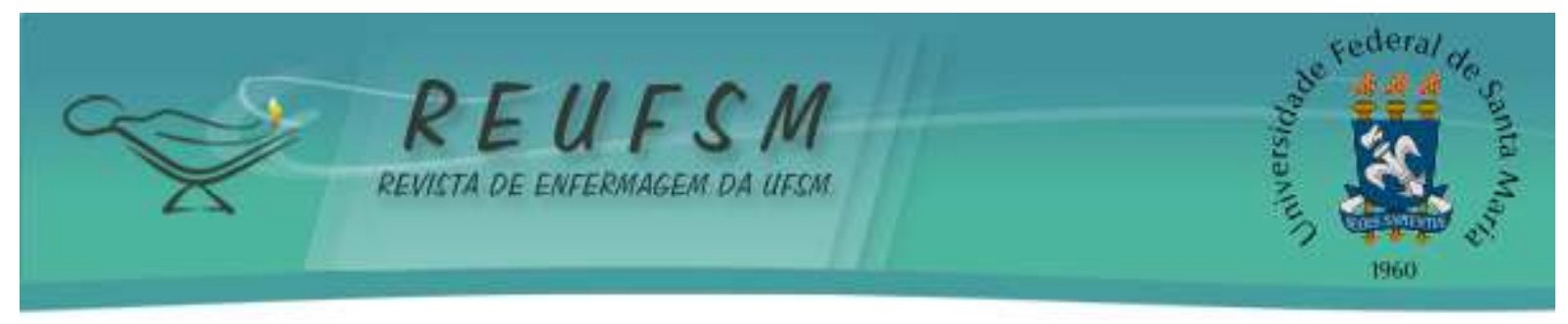

ansiedade, liberdade, ambivalência, medo, culpa e fé. Apesar das dificuldades, buscam força e fé para lutar e manter-se na espera por um doador. ${ }^{25}$ É provável que promover a manifestação de esperança facilitaria a adaptação da pessoa com DRC e seus familiares ao tratamento.

\section{CONCLUSÃO}

A esperança manifestada em pessoas em HD e sua relação com a resiliência, apresentaram correlação positiva e significante, ou seja, quanto maior esperança, mais resiliente é o indivíduo; assim como apresentaram diferenças nas pontuações quando avaliadas segundo sexo, ter alguém que o ajude no cuidado e estar ou não na lista de transplante.

O enfermeiro é agente no processo de desenvolvimento da esperança e resiliência da pessoa em tratamento hemodialítico, oferecendo subsídios de compreensão sobre a doença, a fim de que o paciente com DRC torne-se corresponsável pelo seu tratamento, e que o impulsione para mudanças de comportamento em relação ao seu estilo de vida. Assim, espera-se que os resultados desse estudo possam contribuir para o desenvolvimento de estratégias que colaborem para aperfeiçoar o cuidado de enfermagem ao paciente com doença crônica que está em processo de hemodiálise.

Um aspecto importante a ressaltar é que a esperança e a resiliência apresentaram relação com o apoio familiar. Futuras pesquisas poderiam estudar a contribuição de contar com alguém para o cuidado, a participação em grupos de apoio para pacientes com HD e seus familiares, os quais seriam úteis para promover sua resiliência ao longo do processo da doença e dos tipos de tratamento.

Como limitações do presente estudo, destacamos o tamanho da amostra. O número de participantes corresponde ao de dois centros de diálise, bem como o tempo de tratamento e idade por apresentaram uma ampla variação. Futuros estudos contemplando uma amostra maior poderão, além de estudar as diferenças nesta população, identificar se há diferenças estatisticamente significativas entre esperança e resiliência segundo variáveis estudadas no presente estudo e outras consideradas relevantes. Ainda, o desenvolvimento de estudos com delineamento longitudinal permitiria a identificação de variáveis que possam se apresentar como preditoras para esperança e resiliência em pessoas com DRC e em que etapa da doença as ações de enfermagem poderiam contribuir de forma efetiva. 


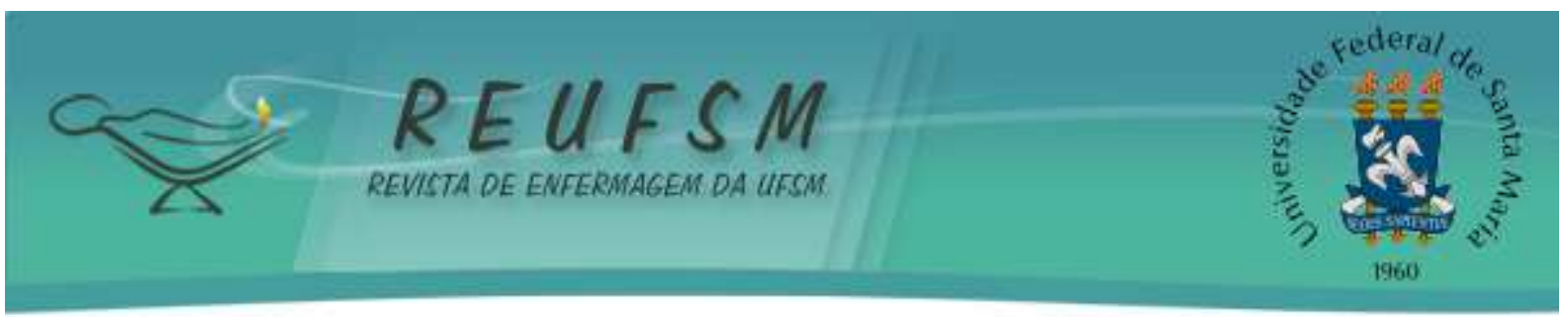

\section{REFERÊNCIAS}

1. World Health Organization. Doenças crônicas não transmissíveis causam 16 milhões de mortes prematuras todos os anos. Genebra: Christian Lindmeier; 2015.

2. Malta DC, Stopal SR, Szwarcwald CL, Gomes NL, Silva Júnior JB, Reis AAC. Surveillance and monitoring of major chronic diseases in Brazil - National Health Survey, 2013. Rev Bras Epidemiol [Internet]. 2015 [acesso em 2016 jun 30]. Disponível em: http://www.scielo.br/pdf/rbepid/v18s2/1980-5497-rbepid-18-s2-00003.pdf.

3. Sesso RC, Lopes AA, Thomé FS, Lugon JR, Martins CT. Inquérito Brasileiro de Diálise Crônica 2014. J Bra Nefrol [Internet]. 2016 [acesso em 2016 jul 02]. Disponível em: http://www.scielo.br/pdf/jbn/v38n1/0101-2800-jbn-38-01-0054.pdf.

4. Tonelli M, Riella M. Chronic kidney disease and the aging population. J Bra Nefrol [Internet]. 2014 [acesso em 2017 maio 15];36(1):1-5. Disponível em: http://www.scielo.br/scielo.php?script=sci_arttext\&pid=S0101-28002014000100001.

5. Fritsch FR, Mayer BLD, Ubessi LD, Kirchner RM, Barbosa DA, Stumm EMF. Atividade física, de lazer e avaliação da saúde na perspectiva de usuários em hemodiálise. Rev Cienc Cuid [Internet]. 2015 [acesso em 2017 maio 31]. Disponível em: http://www.redalyc.org/html/5057/505750948011/.

6. Silva PLN, Oliveira RS, Prates FC, Sena CC, Prates DC, Souto SGT. Prevalência de infecções em cateter de duplo lúmen em um serviço de nefrologia. Rev Enferm UFPE [Internet]. 2014 [acesso em 2017 maio 18];8(7):1882-7. Disponível em: https://periodicos.ufpe.br/revistas/revistaenfermagem/article/view/9862/10087.

7. Araújo JB, Souza Neto VL, Anjos EU, Silva BCO, Rodrigues IDCV, Costa CS. Chronic renal patients everyday on hemodialysis: expectations, modifications and social relations. $\mathbf{J}$ Res Fundam Care Online [Internet]. 2016 [acesso em 2017 maio 18];8(4): 4996-5001. Disponível

em: http://www.seer.unirio.br/index.php/cuidadofundamental/article/view/4404/pdf_1.

8. Orlandi FS, Pepino BG, Pavarini SCI, Santos DA, Mendiondo MSZ. Avaliação do nível de esperança de vida de idosos renais crônicos em hemodiálise. Rev Esc Enferm USP [Internet]. 2012 [acesso em 2016 jun 02];46(4):900-5. Disponível em: http://www.scielo.br/pdf/reeusp/v46n4/17.pdf.

9. Rudnicki T. Doença renal crônica: vivência do paciente em tratamento de hemodiálise. Contextos Clin [Internet]. 2014 [acesso em 2017 jun 10]:7(1):105-16. Disponível em: http://pepsic.bvsalud.org/pdf/cclin/v7n1/v7n1a11.pdf.

10. Rutter M. Psychosocial resilience and protective mechanisms. Am J Orthopsychiatr [Internet]. 1987 [acesso em 2016 jun 29];57(3):316-31. Disponível em: http://psycnet.apa.org/buy/2013-42839-001.

11. Hjemdal O, Friborg O, Stiles TC. Resilience is a good predictor of hopelessness even after accounting for stressful life events, mood and personality (NEO-PI-R). Scand J Psychol [Internet]. 2012 [acesso em 2017 jul 02];53(2). Disponível em: http://onlinelibrary.wiley.com/doi/10.1111/j.1467-

9450.2011.00928.x/abstract;jsessionid=CB2C91B7341199984EA70152A42ABD88.f03t02.

12. Herth K. Development and refinement of an instrument to measure hope. Sch Inq Nurs Pract. 1991[acesso em 2017 jul 02]:5(1):39-51. Disponível em: https://www.ncbi.nlm.nih.gov/pubmed/2063043.

13. Sartore AC, Grossi SAA. Escala de Esperança de Herth: instrumento adaptado e validado 


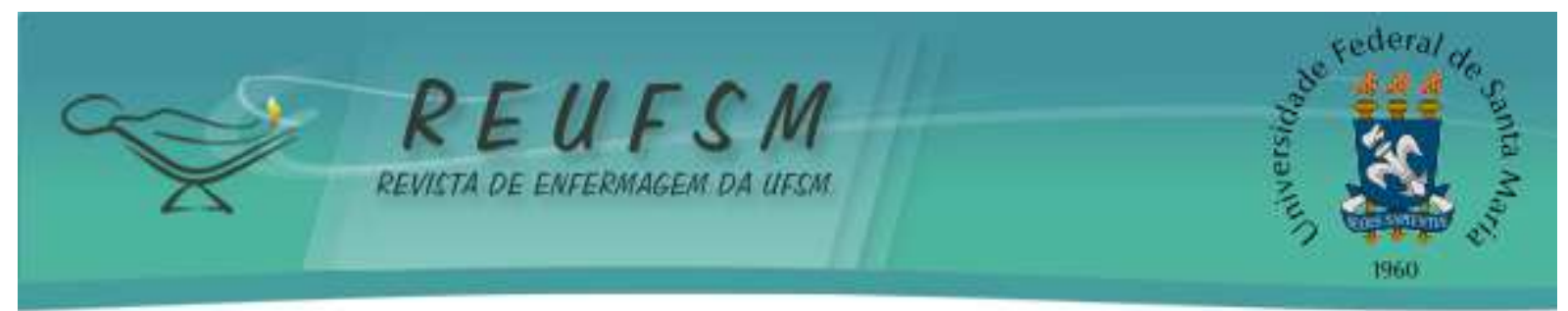

para a língua portuguesa. Rev Esc Enferm USP. 2008 [acesso em 2017 jun 30]:42(2):227-32. Disponível em: https://www.revistas.usp.br/reeusp/article/viewFile/41728/45343.

14. Connor K, Davidson J. Development of a new resilience scale: the Connor-Davidson Resilience Scale (CD-RISC). Anxiety [Internet]. 2003 [acesso em 2016 jul 10];18(2):76-82. Disponível em: https://www.ncbi.nlm.nih.gov/pubmed/12964174.

15. Balsanelli A, Grossi S, Herth K. Avaliação da esperança em pacientes com doença crônica e em familiares ou cuidadores. Acta Paul Enferm [Internet]. 2011 [acesso em 2017 maio 16];24(3):354-8. Disponível

em:

http://www.scielo.br/scielo.php?script=sci_arttext\&pid=S0103-21002011000300008.

16. Salomé G, Alves S, Costa V, Pereira V, Ferreira L. Feelings of powerlessness and hope for cure in patients with chronic lower-limb ulcers. J Wound Care [Internet]. 2013 [acesso em 2017 maio 18]; 22(6):300,302-4. Disponível em: https://www.ncbi.nlm.nih.gov/pubmed/24049812.

17. Shamsalinia A, Pourghaznein T, Parsa M. The relationship between hope and religious coping among patients with type 2 Diabetes. Glob J Health Sci [Internet]. 2015 [acesso em 2016 jun 30];8(1):208-16. Disponível em: https://www.ncbi.nlm.nih.gov/pubmed/26234981.

18. Kavradim S, Özer Z, Bozcuk H. Hope in people with cancer: a multivariate analysis from Turkey. J Adv Nurs [Internet]. 2012 [acesso em 2017 jul 20];69(5):1183-96. Disponível em: https://www.ncbi.nlm.nih.gov/pubmed/22891939.

19. Afrooz R, Rahmani A, Zamanzadeh V, Abdullahzadeh F, Azadi A, Faghany S, et al. The nature of hope among iranian cancer patients. Asian Pac J Cancer Prev [Internet]. 2014 [acesso em 2017 maio 18];15(21):9307-12. Disponível em: https://www.ncbi.nlm.nih.gov/pubmed/25422217.

20. Böell J, Silva D, Hegadoren K. Sociodemographic factors and health conditions associated with the resilience of people with chronic diseases: a cross sectional study. Rev Latinoam Enferm [Internet]. 2016 [acesso em 2017 maio 15];24:e2786. Disponível em: https://www.ncbi.nlm.nih.gov/pmc/articles/PMC5016006/pdf/0104-1169-rlae-24-02786.pdf.

21. Gomes ICC, Lanzotti BR, Orlandi SF. Resilience in patients with chronic kidney disease in hemodialysis. Int J Res Med Sci [Internet]. 2017 [acesso em 2017 jun 30];11(5). Disponível em: https://www.waset.org/publications/10007086/resilience-in-patients-withchronic-kidney-disease-in-hemodialysis.

22. Andrade S, Sesso R, Diniz D. Hopelessness, suicide ideation, and depression in chronic kidney disease patients on hemodialysis or transplant recipients. J Bras Nefrol [Internet]. 2015 [acesso em 2017 jun 29];37(1):55-63. Disponível em: https://www.ncbi.nlm.nih.gov/pubmed/25923751.

23. Oliveira JGR, Lopes VB, Cavalcante LFD, Rocha AFB, Silva RM, Brasil CCP. História de vida do paciente renal crônico: da descoberta ao transplante. CIAIQ [Internet]. 2016 [acesso em 2017 maio 18];2:391-5. Disponível em: https://proceedings.ciaiq.org/index.php/ciaiq2016/article/download/776/763/.

24. Pauletto MR, Breuter M, Timm AMB, Santos NO, Roso CC, Jacobi CS. Transplante renal: percepção de pacientes em hemodiálise fora da lista de espera. Rev Enferm UFSM [Internet]. 2016 [acesso em 2017 maio 15];6(2):154-63. Disponível em: https://periodicos.ufsm.br/reufsm/article/view/20619.

25. Salimena AMO, Ferreira MR. Lista de espera para o transplante renal: revisão integrativa da literatura. Arq Ciênc Saúde [Internet]. 2014 [acesso em 2016 jul 02];21(2)24-33. 


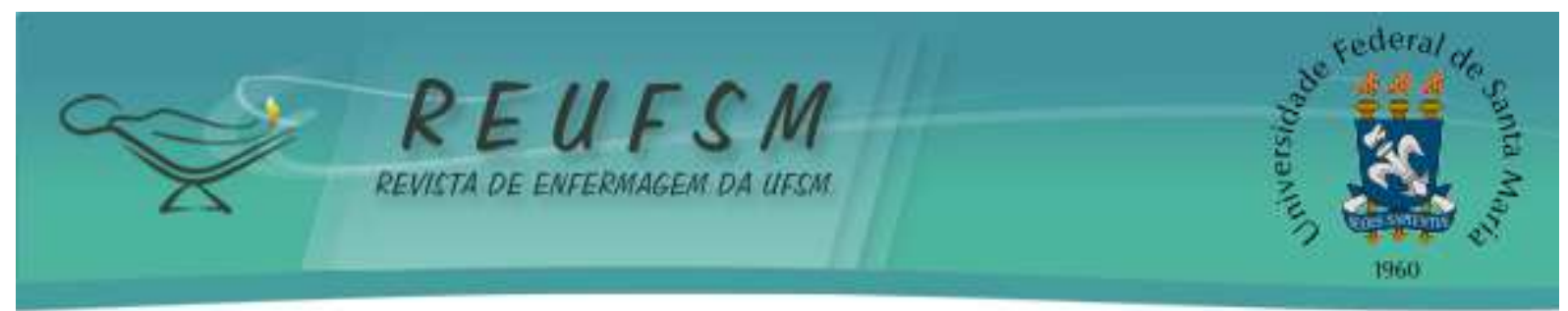

Disponível em: http://repositorio-racs.famerp.br/racs_ol/vol-21-2/02/ID_602_21(2)_Abrjun_2014_-_Revisao.pdf.

Data de submissão: 24/12/2017

Data de aceite: 17/07/2018

Autor correspondente: Carine Ferreira

E-mail: carine2009@hotmail.com

Endereço: Universidade Federal de Santa Catarina. Programa de Pós-graduação em

Enfermagem. Campus Reitor João David Ferreira Lima. Bairro Trindade/Florianópolis/SC.

CEP: 88040-900 